\title{
Contrast effect of emotional context on interpersonal distance with neutral social stimuli
}

Author names: Alice Cartaud ${ }^{\mathrm{a}}$, Vincent Lenglin ${ }^{\mathrm{b}} \&$ Yann Coello $^{\mathrm{a}}$

\section{Affiliations:}

${ }^{a}$ Univ. Lille, CNRS, Lille, UMR 9193 - SCALab - Sciences Cognitives et Sciences Affectives, F-59000 Lille, France

${ }^{\mathrm{b}}$ ETHICS EA 7446, Lille Catholic University, AnthropoLab, Lille, Hauts-de France, France

Running head:

Context effect on interpersonal distance regulation

Corresponding author:

Yann Coello

Laboratoire SCALab UMR CNRS 9193

Université Lille

Domaine universitaire du Pont de Bois, BP 60149

59653 Villeneuve d'Ascq Cedex, France

Email: yann.coello@univ-lille.fr

Phone: +33 320416446 


\begin{abstract}
In social interactions, valence-based judgements are an important component of interpersonal distances regulation. Within the framework of the Range-Frequency model, we tested whether the emotional context, which is known to produce a contrast effect on valence ratings, also contributes to the regulation of interpersonal distances. 51 participants divided in two groups were shown virtual characters with either a neutral facial expression (target-stimuli) or an emotional facial expression (contextual stimuli) in two successive sessions (angry then happy emotional context or vice-versa). The participants rated the valence of the virtual characters and judged the appropriateness of different interpersonal distances. For neutral characters, contrast effect of emotional context was observed, albeit only subtly on interpersonal distance judgments. Overall, the data suggest that although the emotional context influences valence-based judgments of social stimuli, it has a parsimonious effect on interpersonal distance regulation, presumably because the latter relies primarily on emotional facial expression categorical-information.
\end{abstract}




\section{Introduction}

Since Hall (1966), particular attention was paid to the different factors responsible for the natural and automatic regulation of interpersonal distances (IPD) in social contexts, which implies a subtle balance between the need to approach others for efficient social interactions, and the need to maintain a certain margin of safety from others to preserve body integrity (Lloyd, 2009; Siegman \& Feldstein, 2014). Accordingly, a too large IPD would not be appropriate for efficient social interactions, and a too short IPD would trigger discomfort and defensive neurophysiological responses (Cartaud, Ott, Iachini, Honoré, \& Coello, 2020; Cartaud, Ruggiero, Ott, Iachini, \& Coello, 2018; Kennedy, Gläscher, Tyszka, \& Adolphs, 2009; Vieira, Pierzchajlo, \& Mitchell, 2020). Furthermore, egocentric representation of near-body action space (Coello, 2018; Quesque et al., 2017), or others' attributes such as age (Iachini et al., 2016), gender and emotional facial expression (FE) can alter IPD regulation (Ruggiero et al., 2017), highlighting the sensitivity of social spatial regulation to contextual information. As such, emotional FE are crucial cues in social interactions and thus in the regulation of IPD, mainly because they represent a valuable source of information with respect to the emotional state of others (Schrammel, Pannasch, Graupner, Mojzisch, \& Velichkovsky, 2009). Hence, IPD increases when conspecifics display an angry FE whereas it decreases when they display a happy FE (Ruggiero et al., 2017). The regulation of IPD thus correlates with both the strength of the neurophysiological response triggered by a social stimulus when not complying with expected social distances, and the evaluation of its subjective valence (Cartaud et al., 2018). The processing of emotional information conveyed by others, in particular the valence dimension of their FE, seems therefore to be of paramount importance in the regulation of IPD.

A large body of the literature on this matter has shown that judgement (or feeling) of a stimulus' emotional valence depends also on the emotional information conveyed by the context in which the stimulus is embedded (Matsumoto \& Sung Hwang, 2010; Russell \& Fehr, 
1987; Wedell \& Parducci, 1988; Wieser \& Brosch, 2012). As pointed out in different areas of research, judgment of a stimulus refers broadly on relative rather than absolute information, due to the influence of the context (Clark, Frijters, \& Shields, 2008; Furl, 2016; Goldstone, Medin, \& Halberstadt, 1997; Kahneman \& Tversky, 1979; Kontek \& Lewandowski, 2018; Louie, Khaw, \& Glimcher, 2013; Trueblood, Brown, Heathcote, \& Busemeyer, 2013). The notion of context is usually defined along two dimensions: a temporal and a spatial-dimension (Louie, Grattan, \& Glimcher, 2011). The spatial-dimension of the context refers to the set of stimuli presented simultaneously with the target, whereas the temporal-dimension of the context refers to the sequence of stimuli presented across time (Louie et al., 2011). With temporal context, salient information and explicit judgment tasks usually promote contrast effects (Kobylínska \& Karwowska, 2014; Martin, Seta, \& Crelia, 1990), which corresponds to a negative correlation between the judgment of a defined target and the characteristics of the context (Schwarz \& Bless, 2007), that nonetheless decays over time (Louie et al., 2011). As evidence, neutral faces are judged to be more (less) happy when presented in a context made up of negative (positive) faces (Wedell \& Parducci, 1988). Among the models predicting contrast effect, the Range-Frequency (RF) model (Parducci, 1965) proved to be particularly relevant (Wedell, Hayes, \& Kim, 2020; Wedell, Parducci, \& Geiselman, 1987; Wedell \& Parducci, 1988). The RF model is based on the range and frequency principles. According to the range-principle, judgement of a target-stimulus depends on its location relative to the extreme values of the stimuli encountered so far (i.e., the boundaries of the context). According to the frequency-principle, judgement of a target-stimulus depends on the target rank within the distribution of the different values of the stimuli encountered so far. It is assumed that when judging the value of a stimulus, people make a compromise between the range-principle and the frequency-principle (Parducci, 1965).

Although the manipulation of the emotional context, leading to a contrast effect, has 
been extensively investigated in the field of emotional judgment (Wieser \& Brosch, 2012), it is not yet known whether contrast effect can also alter IPD regulation in social context. Our rationale was the following: if preferred IPD is primarily driven by the valence of a social stimulus, thus, altering the valence of that social stimulus by manipulating the emotional context should in turn impact IPD regulation. To address this issue, we designed an online experiment based on RF model simulation (Parducci, 1965). In this experiment, virtual characters with a neutral FE (target-stimuli) were presented singly within a temporal social context made up of characters displaying an emotional FE (happy or angry, contextual stimuli). Contrast effect was evaluated by analyzing the effect of the range and frequency of the contextual emotional characters on valence ratings and IPD judgment of the neutral characters. According to the RF model, in a unimodal context (e.g., negative emotional characters) the perceived valence of the neutral characters should drift towards the opposite direction than the valence of the contextual emotional characters (e.g., a higher positive valence rating). However, in a multimodal context (e.g., negative and positive emotional characters, equally represented across time), the valence attributed to the neutral characters should be repelled by both edges of the contexts, remaining thus close to the central position of the range of the whole set of stimuli. As a corollary, since preferred IPD depends on the subjective valence of the characters, it should be altered by context manipulation. More specifically, preferred IPD with neutral characters embedded in a negative context should be shorter than preferred IPD with neutral characters embedded in a positive context, the effects cancelling each other out in the multimodal presentation of the context. 


\section{Method}

\section{Participants}

Fifty-one adult volunteers completed the entire online experiment (39 women, $\mathrm{M}_{\mathrm{age}}=$ $30.40, \mathrm{SD}_{\text {age }}=9.7, \mathrm{M}_{\text {study }}=4, \mathrm{SD}_{\text {study }}=2.55$ after the French baccalaureate). Based on GPower (version 3.1, within-between ANOVA $\alpha=0.05$, Cohen's F $=0.25$ ), the requested sample size was 36 participants, but we decided to increase the number of participants because the experiment was completed online. All of the participants had normal vision or were invited to use optical correction (e.g., wear glasses), if they consented to take part in the experiment. The protocol received approval by the local institutional ethics committee (2020-426-S83).

\section{Apparatus and stimuli}

The experiment was created on lab.js builder (Henninger, Shevchenko, Mertens, \& Kieslich, 2019), hosted and run online on the CNRS web server. Advertisement was shared on social and professional networks. The stimuli consisted of 14 male and 14 female characters selected from the ATHOS database (Cartaud \& Coello, 2020). Two male and two female characters had a neutral FE (targets), five males and five females had an angry FE and the last five of each gender had a happy FE (context). Both the characters and the empty room in which they were presented were built on Unity (2018.2.21f1 version, Fig. 1).

Depending on the task, the characters were presented at different distances ( 25 to 135 $\mathrm{cm}$ ) from the participants, who had to represent themselves being on the proximal side of the virtual room. The distance increment was $6 \mathrm{~cm}$ from 25 to $85 \mathrm{~cm}$ and was $10 \mathrm{~cm}$ from 85 to $105 \mathrm{~cm}$, thus resulting in a total of 16 different distances. This presentation was used in order to minimize the number of trials while keeping the increment as small as possible, allowing us to experimentally scan a reasonable range of distances. 


\section{Procedure and design}

First, participants completed a short questionnaire focusing on general information (age, gender, study level). Then, in two sessions, participants performed a set of three tasks twice (Fig. 1). Each participant was randomly assigned to one of two groups that differed only in the order of presentation of the emotional context. For group $\mathrm{AH}(\mathrm{N}=23)$, the emotional context of the first session consisted of angry characters (negative unimodal context) and that of the second session consisted of happy characters (positive and multimodal context due to previous exposition to the other emotional context). The pattern was reversed for group HA $(\mathrm{N}=28$, session 1 consisted of happy contextual characters and session 2 consisted of angry contextual characters). For each participant, the pair of neutral characters (one male and one female) was randomly assigned to each session.

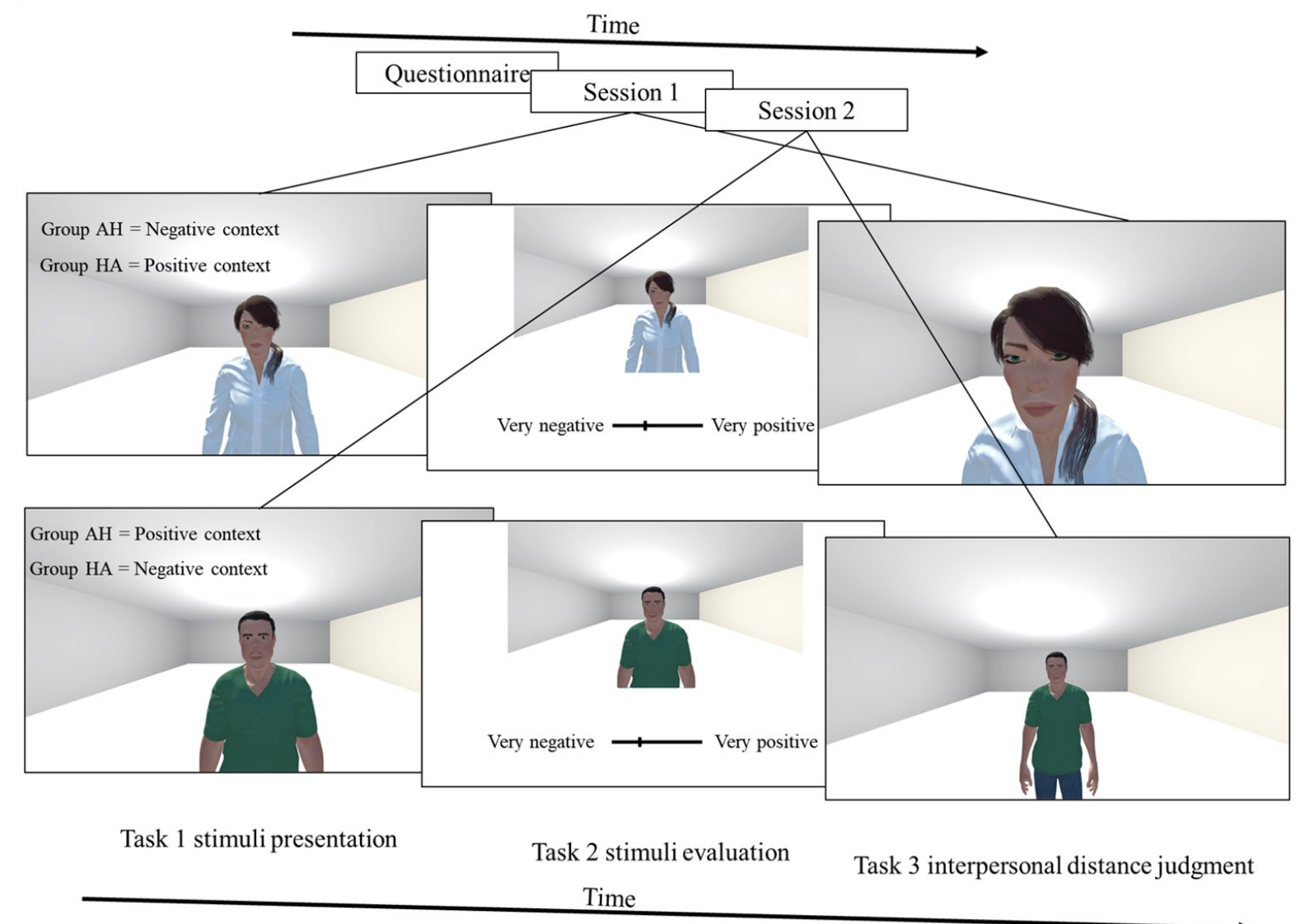

Fig. 1. Schematic representation of the experimental course (top panel) and sessions (bottom panel). 
In the first task, the context was settled up using 10 emotional characters (happy or angry depending on the group and the session) and 2 neutral characters (one of each gender). During the first session, since (i) the neutral stimuli were at the edges of the range in terms of valence (neutral characters have either the minimum or the maximum valence rating depending on the context) and (ii) the distributions of the stimuli were skewed (context was either mostly negative or mostly positive), the RF model predicted a strong contrast effect (Parducci, 1965). Participants passively but carefully observed successive pictures of the characters. Each character was presented twice in a random order for 2 secs at $61 \mathrm{~cm}$ from the proximal side of the room in full screen mode. A white screen was displayed for $500 \mathrm{~ms}$ between each character presentation.

In the second task, participants sequentially evaluated the valence of the two neutral characters and two contextual emotional characters (one male and one female) which corresponded to the stimuli that received the highest positive or negative valence according to the ATHOS database (Cartaud \& Coello, 2020). The number of evaluations of contextual characters was limited in order to prevent the online experiment extending too much in time (reducing thus the number of dropouts). The characters were displayed at $61 \mathrm{~cm}$ from the proximal side of the room and the picture filled $60 \%$ of the computer screen. Underneath each character was positioned a slider question for the evaluation of the valence of the character. Participants had to position a cursor along the horizontal line using the computer trackpad or a mouse (10 units) with the labels "very negative" on the left side and "very positive" on the right side of the slider (see Fig. 1). No numerical feedback of their rating was provided.

The third task consisted in judging whether the distance between the participants and each character was appropriate for social interaction or not (IPD judgment). The same characters as in the previous task (two neutral target-characters and two contextual emotional characters) were presented singly and randomly at a distance varying from 25 to $135 \mathrm{~cm}$. 
Participants had to respond as fast and as spontaneously as possible by pressing the "L" (appropriate) or "S" (inappropriate) keys of their keyboard. The characters remained on the screen until the participant's response was provided, then followed by a white screen lasting 500 ms. Each character pseudo-randomly appeared four times at each distance (Character's gender [2] * FE [2] * Distance [16] * Repetition [4]), leading to a total of 256 trials. A break was possible following the completion of the first 128 trials. Participants started with a 6 -trials training session with characters that were not used in the main experiment, in order to operationalize the association between responses and computer keys.

Following the first (unimodal) session, a break was proposed before launching the second (multimodal) session, which consisted of the same three tasks, but with another emotional context and two new characters displaying a neutral FE. Thus, during the second session, the contextual characters had a happy FE for group AH and an angry FE for group HA.

\section{Control task}

A control task was designed to quantify the contextless rating of the neutral characters in terms of valence. During this task, another group of 20 participants performed a valence rating of the neutral characters used in the main experiment. One of them was discarded because he/she didn't move the sliders while completing the evaluation $(\mathrm{N}=19,9$ women, Mage $=$ 26.16, $\mathrm{SDage}=8.62, \mathrm{M}_{\text {study }}=4, \mathrm{SD}_{\text {study }}=2.59$ after the French baccalaureate). The characters assessed consisted of the four target-characters with the neutral FE (two males and two females). As in the main experiment, the characters were first sequentially presented, then participants rated their valence as in task 2 (varying from very negative to very positive).

\section{Data analysis}

Statistical analyses were carried out using Bayesian linear mixed models' regressions (Bürkner, 2017; Gelman, Lee, \& Guo, 2015), with R (version 4.0.2) and R Studio software 
(version 1.3.1056). The main independent variables of interest were the Stimulus FE (angry, happy, neutral), the Context (negative, positive), the Session (unimodal, multimodal), and their combination. We focused on the contrast between eight combinations: angry negative unimodal (or multimodal), happy positive unimodal (or multimodal), neutral negative unimodal (or multimodal), and neutral positive unimodal (or multimodal). More specifically for every task, we analyzed the contrasts between:

- Neutral negative unimodal - neutral positive unimodal (unimodal context effect)

- Neutral negative multimodal - neutral positive multimodal (multimodal context effect)

- Neutral negative (positive) unimodal - neutral negative (positive) multimodal (context effect across the sessions)

In addition, we compared the valence ratings of the neutral stimuli (negative and positive context, unimodal and multimodal session) to that of the control group to quantify the effect of the emotional context. Finally, we tested for the Gender effect of both the participants and the characters in the IPD judgment task, but controlled these effects for the unimodal, multimodal and across sessions analysis of the valence ratings.

For every model, we considered interindividual variability by adding the Subject level random effect. We specified a mildly informative normal distribution on the fixed effects (constraining the magnitude of the effects to reasonable values without specific direction) and used the default brms priors for the random effects when applicable. The approximation of the posterior distribution was obtained through 8000 Markov chain Monte Carlo (4 chains of each 2000 warm-up samples), whose convergence was validated through visual inspection and by computing the Rhat statistic. The Credible Intervals (CI) were fixed at 95\% to judge contrasts between estimates of the parameters of the posterior distribution as probable. We performed linear regressions with a normal distribution for the analyses of the valence ratings and a logistic 
regression with a binomial distribution likelihood for the analysis of IPD.

For each condition, the preferred IPD were determined from the participants' appropriate/inappropriate responses at each distance using the following equation:

$$
y=\frac{1}{1+e^{-(\alpha+\beta X)}}
$$

where $y$ is the participants' probability to answer "appropriate", and $X$ is the distance. From this equation, we computed the ratio $-\alpha / \beta$ giving the critical value of $X$ corresponding to the transition between the appropriate and inappropriate responses (i.e., the inflection point of the logistic function). This value is interpreted as the preferred IPD. The slope at the vicinity of the inflection point was retrieved from $\beta / 4$. Data and statistical analysis are available on the OSF platform (https://osf.io/dur4k/?view_only=0199de84522546cf96289c434f7e928f).

\section{Results}

For every task, estimates and their $95 \% \mathrm{CI}$ are reported in Table 1 and the contrasts of interest listed in the data analysis section are reported in Table 2. The slopes of the logistic regressions were not statistically different across the conditions suggesting that the different conditions tested had the same level of difficulty (slope difference between the neutral characters from the negative and the positive context in the unimodal session: $0.02[-0.51 ; 0.54]$ and in the multimodal session: $-0.22[-0.74 ; 0.31])$. 
Table 1. Posterior estimates and $95 \%$ CI for the dependent variables analyzed: valence ratings $(0-10$ scale) in the experimental and control groups, preferred IPD (cm) and its slope (at IPD threshold) as a function of Stimulus FE, Context, Session, Participants' Gender, and Characters' Gender.

\begin{tabular}{|c|c|c|c|c|c|}
\hline Dependent variable & Stimulus & Context & Session & Mean & $95 \% \mathrm{CI}$ \\
\hline \multirow[t]{8}{*}{$\begin{array}{l}\text { Experimental group } \\
\text { Valence ratings }\end{array}$} & Angry & Negative & Unimodal & 0.85 & {$[0.51 ; 1.2]$} \\
\hline & Angry & Negative & Multimodal & 1.51 & {$[1 ; 1.99]$} \\
\hline & Happy & Positive & Unimodal & 7.63 & {$[7.21 ; 8.05]$} \\
\hline & Happy & Positive & Multimodal & 7.95 & {$[7.27 ; 8.61]$} \\
\hline & Neutral & Negative & Unimodal & 6.41 & {$[5.85 ; 6.96]$} \\
\hline & Neutral & Negative & Multimodal & 5.31 & {$[4.93 ; 5.7]$} \\
\hline & Neutral & Positive & Unimodal & 2.81 & {$[2.25 ; 3.38]$} \\
\hline & Neutral & Positive & Multimodal & 4.56 & {$[4.02 ; 5.1]$} \\
\hline $\begin{array}{l}\text { Control group } \\
\text { Valence ratings } \\
\end{array}$ & Neutral & Control & & 4.59 & {$[4.04 ; 5.15]$} \\
\hline \multirow[t]{12}{*}{ Preferred IPD } & Angry & Negative & Unimodal & 58.51 & {$[50.95 ; 67.36]$} \\
\hline & Angry & Negative & Multimodal & 60.75 & {$[51.39 ; 72.4]$} \\
\hline & Happy & Positive & Unimodal & 47.78 & {$[42.13 ; 54.53]$} \\
\hline & Happy & Positive & Multimodal & 44.38 & {$[38.22 ; 51.67]$} \\
\hline & Neutral & Negative & Unimodal & 48.39 & {$[40.71 ; 57.19]$} \\
\hline & Neutral & Negative & Multimodal & 46.95 & {$[40.54 ; 54.65]$} \\
\hline & Neutral & Positive & Unimodal & 53.33 & {$[46.67 ; 61.17]$} \\
\hline & Neutral & Positive & Multimodal & 50.44 & {$[43.09 ; 59.02]$} \\
\hline & $\begin{array}{l}\text { Male } \\
\text { Participants }\end{array}$ & & & 54.86 & {$[45.71 ; 65.44]$} \\
\hline & $\begin{array}{l}\text { Female } \\
\text { Participant }\end{array}$ & & & 50.34 & {$[44.53 ; 57.36]$} \\
\hline & Male Characters & & & 52.09 & {$[46.39 ; 59.03]$} \\
\hline & Female Character & & & 50.72 & {$[45.32 ; 57.17]$} \\
\hline \multirow[t]{8}{*}{$\begin{array}{l}\text { Slope of preferred } \\
\text { IPD }\end{array}$} & Angry & Negative & Unimodal & 3.9 & {$[3.31 ; 4.47]$} \\
\hline & Angry & Negative & Multimodal & 3.64 & {$[2.98 ; 4.29]$} \\
\hline & Happy & Positive & Unimodal & 4.44 & {$[3.82 ; 5.04]$} \\
\hline & Happy & Positive & Multimodal & 4.79 & {$[4.12 ; 5.45]$} \\
\hline & Neutral & Negative & Unimodal & 4.16 & {$[3.53 ; 4.82]$} \\
\hline & Neutral & Negative & Multimodal & 4.16 & {$[3.53 ; 4.8]$} \\
\hline & Neutral & Positive & Unimodal & 4.15 & {$[3.54 ; 4.75]$} \\
\hline & Neutral & Positive & Multimodal & 4.38 & {$[3.74 ; 5.04]$} \\
\hline
\end{tabular}


Table 2. Context effects between the estimates and 95\% CI for every dependent variable evaluated: valence ratings (experimental group, control group) and preferred IPD (cm). Post-hoc analysis is also reported for IPD threshold (Emotion effect).

\begin{tabular}{|c|c|c|c|c|c|}
\hline $\begin{array}{l}\text { Dependent } \\
\text { variable } \\
\end{array}$ & Effect & Estimate 1 & Estimate 2 & Contrast & $95 \%$ CI \\
\hline \multirow[t]{4}{*}{$\begin{array}{l}\text { Experimental group } \\
\text { Valence ratings }\end{array}$} & Unimodal & $\begin{array}{l}\text { Neutral } \\
\text { Negative } \\
\text { Unimodal }\end{array}$ & $\begin{array}{l}\text { Neutral Positive } \\
\text { Unimodal }\end{array}$ & 3.6 & {$[2.81 ; 4.39]$} \\
\hline & Multimodal & $\begin{array}{l}\text { Neutral } \\
\text { Negative } \\
\text { Multimodal }\end{array}$ & $\begin{array}{l}\text { Neutral Positive } \\
\text { Multimodal }\end{array}$ & 0.75 & {$[0.09 ; 1.42]$} \\
\hline & \multirow[t]{2}{*}{$\begin{array}{l}\text { Across } \\
\text { sessions }\end{array}$} & $\begin{array}{l}\text { Neutral } \\
\text { Negative } \\
\text { Unimodal }\end{array}$ & $\begin{array}{l}\text { Neutral Negative } \\
\text { Multimodal }\end{array}$ & 1.1 & {$[0.42 ; 1.77]$} \\
\hline & & $\begin{array}{l}\text { Neutral Positive } \\
\text { Unimodal }\end{array}$ & $\begin{array}{l}\text { Neutral Positive } \\
\text { Multimodal }\end{array}$ & -1.75 & {$[-2.52 ;-0.96]$} \\
\hline \multirow[t]{4}{*}{$\begin{array}{l}\text { Control group } \\
\text { Valence ratings }\end{array}$} & \multirow[t]{4}{*}{$\begin{array}{l}\text { Emotional } \\
\text { context }\end{array}$} & $\begin{array}{l}\text { Neutral } \\
\text { Negative } \\
\text { Unimodal }\end{array}$ & Neutral Control & 1.81 & {$[1.01 ; 2.61]$} \\
\hline & & $\begin{array}{l}\text { Neutral } \\
\text { Negative } \\
\text { Multimodal }\end{array}$ & Neutral Control & 0.64 & {$[-0.1 ; 1.39]$} \\
\hline & & $\begin{array}{l}\text { Neutral Positive } \\
\text { Unimodal }\end{array}$ & Neutral Control & -1.74 & {$[-2.58 ;-0.94]$} \\
\hline & & $\begin{array}{l}\text { Neutral Positive } \\
\text { Multimodal } \\
\end{array}$ & Neutral Control & -0.02 & {$[-0.83 ; 0.8]$} \\
\hline \multirow[t]{11}{*}{ Preferred IPD } & Unimodal & $\begin{array}{l}\text { Neutral } \\
\text { Negative } \\
\text { Unimodal }\end{array}$ & $\begin{array}{l}\text { Neutral Positive } \\
\text { Unimodal }\end{array}$ & -4.94 & {$[-13.26 ; 3.44]$} \\
\hline & Multimodal & $\begin{array}{l}\text { Neutral } \\
\text { Negative } \\
\text { Multimodal }\end{array}$ & $\begin{array}{l}\text { Neutral Positive } \\
\text { Multimodal }\end{array}$ & -3.49 & {$[-11.61 ; 4.53]$} \\
\hline & \multirow[t]{2}{*}{$\begin{array}{l}\text { Across } \\
\text { sessions }\end{array}$} & $\begin{array}{l}\text { Neutral } \\
\text { Negative } \\
\text { Unimodal }\end{array}$ & $\begin{array}{l}\text { Neutral Negative } \\
\text { Multimodal }\end{array}$ & 1.44 & {$[-6.85 ; 9.98]$} \\
\hline & & $\begin{array}{l}\text { Neutral Positive } \\
\text { Unimodal }\end{array}$ & $\begin{array}{l}\text { Neutral Positive } \\
\text { Multimodal }\end{array}$ & 2.89 & {$[-5.16 ; 10.76]$} \\
\hline & \multirow[t]{2}{*}{ Gender } & $\begin{array}{l}\text { Male } \\
\text { Participants }\end{array}$ & $\begin{array}{l}\text { Female } \\
\text { Participants }\end{array}$ & 4.52 & {$[-4.43 ; 14.14]$} \\
\hline & & $\begin{array}{l}\text { Male } \\
\text { Characters }\end{array}$ & $\begin{array}{l}\text { Female } \\
\text { Characters }\end{array}$ & 1.37 & {$[0.67 ; 2.14]$} \\
\hline & \multirow[t]{4}{*}{$\begin{array}{l}\text { Emotion } \\
\text { (post-hoc) }\end{array}$} & $\begin{array}{l}\text { Angry Negative } \\
\text { Unimodal }\end{array}$ & $\begin{array}{l}\text { Neutral Negative } \\
\text { Unimodal }\end{array}$ & 10.13 & {$[3.98 ; 16.19]$} \\
\hline & & $\begin{array}{l}\text { Happy Positive } \\
\text { Unimodal }\end{array}$ & $\begin{array}{l}\text { Neutral Positive } \\
\text { Unimodal }\end{array}$ & -5.55 & {$[-9.16 ;-2.12]$} \\
\hline & & $\begin{array}{l}\text { Angry Negative } \\
\text { Unimodal }\end{array}$ & $\begin{array}{l}\text { Neutral Positive } \\
\text { Unimodal }\end{array}$ & 5.18 & {$[-2.17 ; 12.68]$} \\
\hline & & $\begin{array}{l}\text { Happy Positive } \\
\text { Unimodal }\end{array}$ & $\begin{array}{l}\text { Neutral Negative } \\
\text { Unimodal }\end{array}$ & -0.61 & {$[-8.73 ; 7.39]$} \\
\hline & Valence & & & -0.82 & {$[-1.23 ;-0.43]$} \\
\hline
\end{tabular}

Note: Contrasts with CIs that do not overlap with zero are in italics (i.e., robust estimate with $95 \% \mathrm{CI}$ ). 


\section{Valence ratings}

The main effects observed on valence ratings during the experiment are represented in Fig. 2. The Bayes R-Squared of the fit for the valence ratings was computed using the function bayes_R2 and revealed a coefficient of $0.85[0.83 ; 0.87]$. Contrast effects were observed: during the unimodal session, the neutral characters embedded in the negative context were judged as more positive than the neutral characters embedded in the positive context $(+3.6[2.81 ; 4.39])$. This effect was much smaller during the multimodal session $(+0.75[0.09 ; 1.42])$. In line with these results, valence ratings of the neutral characters in the unimodal session were more pronounced than in the multimodal session (difference in negative context: $1.1[0.42 ; 1.77]$; difference in positive context: $-1.75[-2.52 ;-0.96])$.

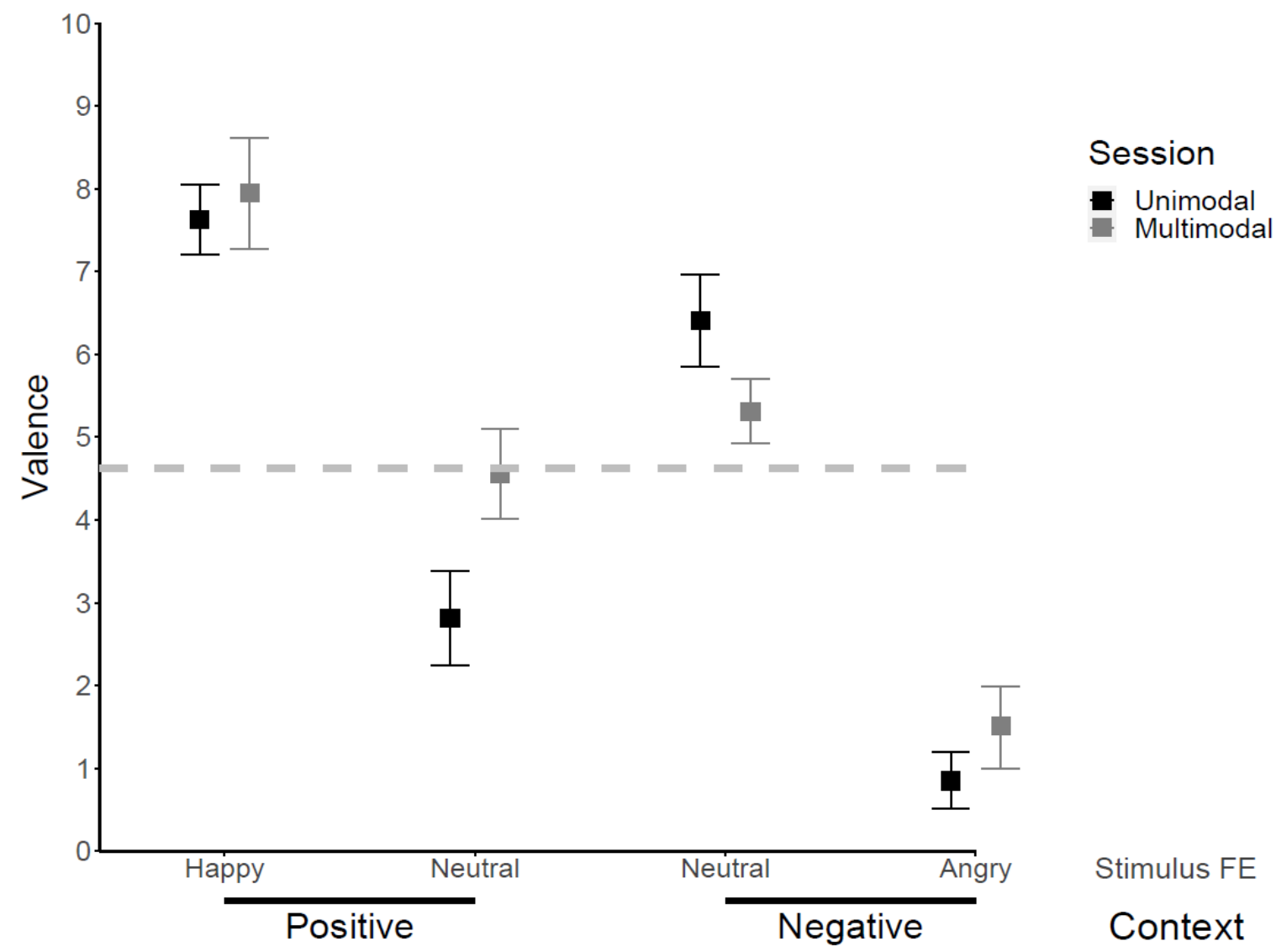

Fig. 2. Graphical representation of the Posterior Mean Valence and the 95\% CI as a function of Stimulus FE, Context and Session. Grey horizontal line represents the average rating of neutral targets obtained by the control group. 
Finally, we compared valence ratings obtained for neutral characters during the experiment to those obtained in the control group (Fig. 3, averaged Bayes $R^{2}=0.54[0.45 ; 0.6]$ ). As presented in Table 2, only valence ratings performed in the unimodal session differed from the ratings provided by the control group.

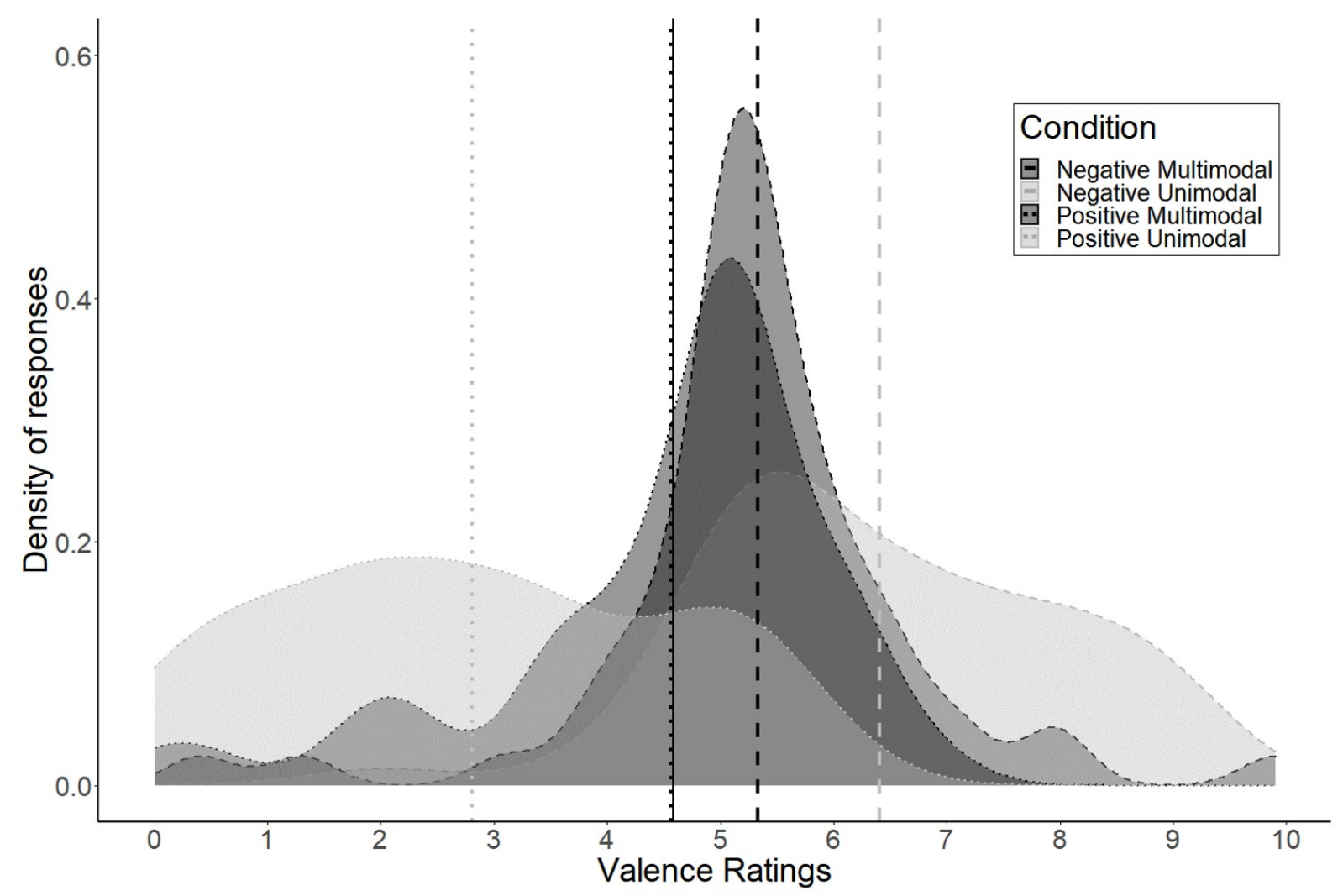

Fig. 3. Density of Responses Relating to Valence Ratings for the neutral characters in the experimental group (dashed grey line: negative unimodal context; dotted grey line: positive unimodal context; dashed dark line: negative multimodal context; dotted dark line: positive multimodal context). Dashed and dotted grey vertical lines represent the average rating of neutral characters in the positive and negative context, respectively (unimodal session). Dashed and dotted dark vertical lines represent the average rating of neutral characters in the positive and negative context, respectively (multimodal session). The solid vertical dark line represents the average valence ratings obtained by the control group.

\section{Interpersonal distances}

Concerning the analysis of IPD judgments and as reported in Table 2, valence ratings drove preferred IPD since each 1-point increase in valence ratings led to a decrease in IPD of almost $1 \mathrm{~cm}\left(-0.82 \mathrm{~cm}[-1.23 ;-0.43]\right.$, averaged Bayes $\left.\mathrm{R}^{2}=0.91[0.90 ; 0.91]\right)$. However, and as 
presented in Fig. 4, no contrast effect emerged when comparing preferred IPD with the neutral characters embedded in the positive context with those with the neutral characters embedded in the negative context, neither in the unimodal nor in the multimodal session.

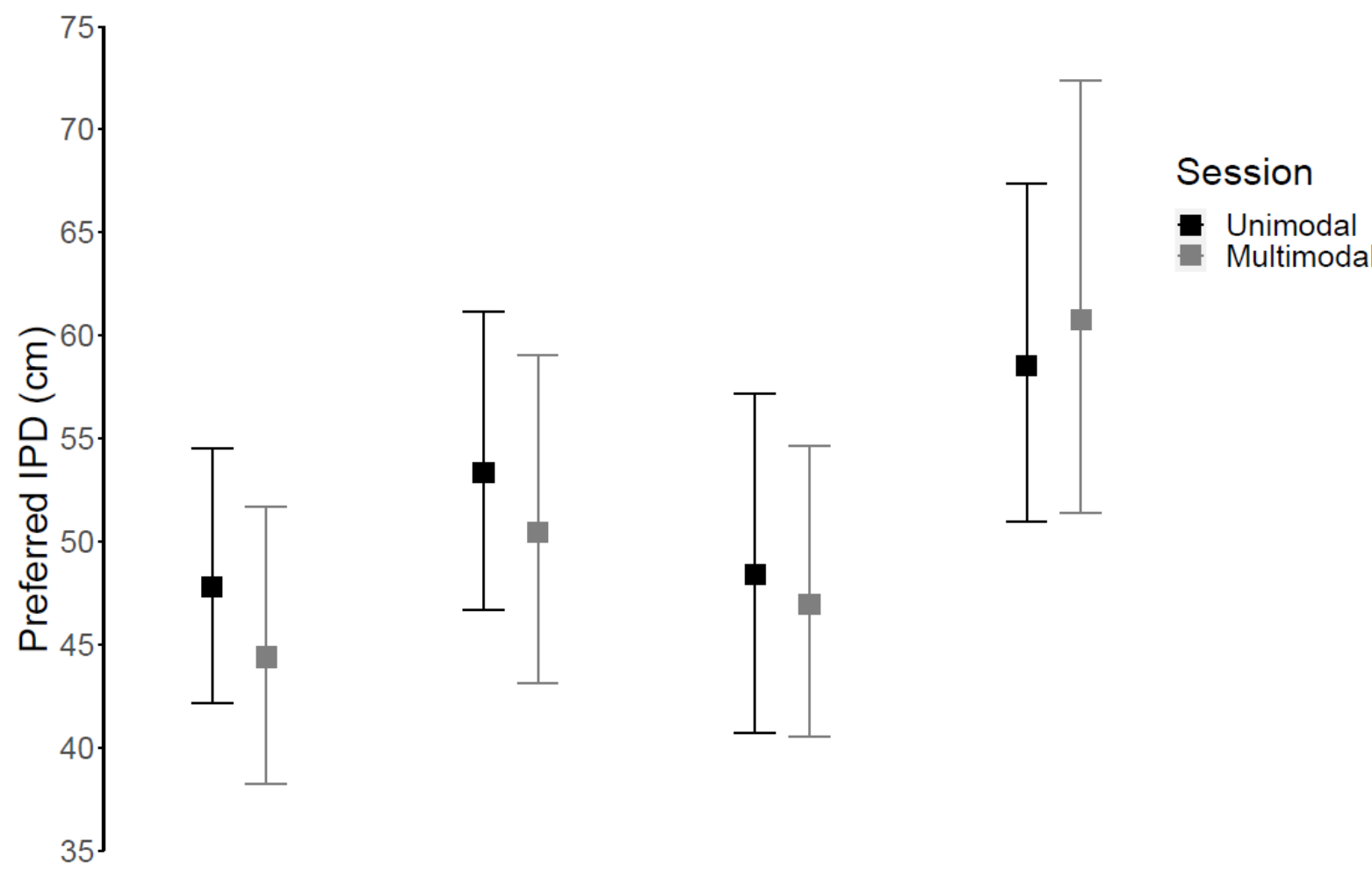

\begin{tabular}{|c|c|c|c|}
\hline Hарру & Neutral & Neutral & Angry \\
\hline
\end{tabular}

Fig. 4. Mean Prediction and 95\% CI of Posterior distribution of Preferred IPD per stimulus, context and session.

\section{Post-hoc analysis}

To further analyze the contrast effect of the context on IPD regulation, we conducted a post-hoc analysis for the unimodal session only. More precisely, we investigated whether the contrast effect would emerge when comparing preferred IPD with the neutral characters to preferred IPD with the emotional characters in each context (Table 2, see Emotion effect for 
preferred IPD). First, we observed that preferred IPD with the neutral characters differed from preferred IPD with the contextual emotional characters when belonging to the same context. Preferred IPD was larger with angry characters than with neutral characters embedded in the same negative context $(+10.13 \mathrm{~cm},[3.98 ; 16.19])$, whereas preferred IPD was shorter with happy characters than with neutral characters embedded in the same positive context $(-5.55 \mathrm{~cm}$ $[-9.16 ;-2.12])$. Second and appealingly, preferred IPD with the neutral characters did not differ from preferred IPD with the contextual emotional characters when belonging to different contexts. More precisely, preferred IPD with angry characters was not different from that with neutral characters embedded in the positive context $(+5.18 \mathrm{~cm}[-2.17 ; 12.68])$. Similarly, preferred IPD with happy characters was not different from preferred IPD with neutral characters embedded in the negative context $(-0.61 \mathrm{~cm}[-8.73 ; 7.39])$. Therefore, even though the contrast effect was not directly observed on IPD regulation when comparing preferred IPD with the neutral characters of each context, the above analysis revealed that the emotional context specifically altered preferred IPD with the neutral characters.

\section{Discussion}

In social context, IPD regulation is dependent on the emotional state perceived in others: the more conspecifics seem positive, the shorter the preferred IPD; conversely the more conspecifics seem negative, the larger the preferred IPD (Ruggiero et al., 2017). However, it has been highlighted that judging the affective dimension in others also depends on the emotional context (Wedell \& Parducci, 1988). More precisely, a contrast effect (i.e., a negative correlation between the valence ratings of a neutral target and the valence of the context), which can be formalized by the RF model, has been often reported (Wedell \& Parducci, 1988). The aim of the present study was to establish whether the valence of a neutral character, when influenced by an emotional context (using positive and negative emotional characters), leads 
also to congruent IPD regulation.

On the one hand, and in line with previous studies, we observed a contrast effect on valence ratings of neutral characters when presented in the unimodal session. Thus, neutral characters were judged more positively when presented in a context made up with angry characters than when presented in a context made up with happy characters (Wedell \& Parducci, 1988). On the other hand, when participants were exposed to an initial emotional context (e.g., positive), then to a contrasting emotional context (e.g., negative), valence ratings of the neutral characters were influenced by both contexts (Parducci, 1965). Indeed, the average valence ratings was 2.81 (6.41) for the neutral characters embedded in the positive (negative) context of the unimodal session, whereas it was 4.56 (5.31) for the neutral characters embedded in the positive (negative) context of the multimodal session, which did not differ from the control group (4.59, a value close to the central position of the range of the whole set of stimuli). This suggests that the same emotional context did not have the same impact on valence ratings of the neutral characters depending on whether or not it was preceded by a contrasting emotional context. Accordingly, valence ratings of the neutral characters embedded in a negative multimodal context differed only slightly from those of the neutral characters embedded in a positive multimodal context. This indicates that both contexts influenced neutral characters judgments, nearly cancelling each other, and suggests the presence of a small temporal decay of the influence of previously encountered contextual stimuli on valence ratings, which was not predicted by the RF model (Parducci, 1965).

With respect to the effect of emotional context on IPD regulation in the unimodal session, preferred IPD with neutral characters was specifically altered by the emotional context in the direction predicted by the contrast effect. Indeed, while preferred IPD with neutral characters (in either the negative or positive context) deviated from preferred IPD with the emotional characters within the same context, it did not differ from preferred IPD with the 
emotional characters of the other context (unimodal session). This result is in accordance with the presence of a contrast effect by showing that preferred IPD with the neutral characters in the positive context shifted towards and eventually became not different from the preferred IPD with the angry characters, while being larger than preferred IPD with the happy characters. A mirror pattern of results was observed when considering the neutral characters in the negative unimodal context. Therefore, the contrast effect produced by the emotional context, which was observed on valence ratings, extended to IPD regulation. Consistent with our hypothesis, as valence ratings of neutral characters increased (decreased) in the negative (positive) emotional context, preferred IPD decreased (increased) with respect to the same neutral characters. These results confirmed, thus, the dependency of IPD regulation on the subjective valence attributed to social stimuli (Coello \& Cartaud, 2021), although going deeper into the underlying processes by highlighting that the emotional context also impacts IPD regulation in social interactions.

The present study however revealed that when directly comparing preferred IPD with the neutral characters embedded in either a positive or negative emotional context, no statistical difference emerged. Indeed, despite the contrast effect was directly observed on valence ratings, this was not the case on IPD judgments, although the difference observed in the latter was in the direction of the contrast effect. This lack of direct observation of the contrast effect on preferred IPD in the unimodal session could be related to the intensity of the emotional stimuli used in the experiment. Although the latter produced a strong contrast effect on valence ratings, the emotional characters may not have been sufficiently valenced to trigger a strong contrast effect on preferred IPD with the same neutral characters. In support of this interpretation, it is worth noting the negative relationship between valence ratings and preferred IPD, which revealed that for one-point gained in valence ratings, preferred IPD decreased by only $0.82 \mathrm{~cm}$. Therefore, variations in the valence scale might have been not broad enough to obtain, with the neutral characters, a substantial change in preferred IPD. This interpretation echoes the 
semantic space of emotion theory (Cowen \& Keltner, 2021). Indeed, one may speculate that IPD regulation is more sensitive to the specific emotional category conveyed by conspecifics than to the subtle fluctuations of their valence dimension induced by the emotional context, weakly relevant for dyads or small group social interaction. Testing different levels of intensity of the emotional context would be necessary in the future to confirm these interpretations (Wieser \& Brosch, 2012). Notwithstanding the constraints inherent in the effect of the intensity of the emotional context on social regulation, comparing the unimodal emotional contexts in the present study provided strong support for the contrast effect. Consequently, the reported findings pave the way for more in-depth investigations into the effect of emotional context on IPD regulation.

Overall, the present study validates the well-known contrast effect of emotional context on valence judgments. As a corollary, changing valence ratings of a neutral social stimulus through the emotional context alters IPD regulation in the direction of a contrast effect although subtly. Further investigations would be needed to decipher whether the effect of emotional context on IPD regulation requires highly emotional context to observe strong contrast effect, or whether IPD regulation is simply poorly sensitive to the emotional context, regardless of its intensity, possibly in relation to the priority given to the emotional category of conspecifics in social interaction. 


\section{References}

Bürkner, P. C. (2017). brms: An R Package for Bayesian Multilevel Models Using Stan. Journal of Statistical Software, 80(1), 1-28. https://doi.org/10.18637/jss.v080.i01

Cartaud, A., \& Coello, Y. (2020). ATHOS: a dATabase of 48 3D Human virtual characters with non-emOtional facial expreSsion for virtual reality. Psyarxiv.Com. https://doi.org/10.31234/osf.io/r7fby

Cartaud, A., Ott, L., Iachini, T., Honoré, J., \& Coello, Y. (2020). The influence of facial expression at perceptual threshold on electrodermal activity and social comfort distance. Psychophysiology, 57(9), e13600. https://doi.org/10.1111/psyp.13600

Cartaud, A., Ruggiero, G., Ott, L., Iachini, T., \& Coello, Y. (2018). Physiological Response to Facial Expressions in Peripersonal Space Determines Interpersonal Distance in a Social Interaction Context. Frontiers in Psychology, 9. https://doi.org/10.3389/fpsyg.2018.00657

Clark, A. E., Frijters, P., \& Shields, M. A. (2008). Relative income, happiness, and utility: An explanation for the Easterlin paradox and other puzzles. Journal of Economic Literature, 46(1), 95-144. https://doi.org/10.1257/jel.46.1.95

Coello, Y. (2018). Action Spaces Representation in Social Contexts. In K. Shigemasu, S. Kuwano, T. Sato, \& T. Matsuzawa (Eds.), Diversity in Harmony - Insights from Psychology (John Wiley \& Sons, pp. 230-254). John Wiley \& Sons, Ltd. https://doi.org/10.1002/9781119362081.ch12

Coello, Y., \& Cartaud, A. (2021). The interrelation between peripersonal action space and interpersonal social space: Psychophysiological evidence and clinical implications. Frontiers in Human Neuroscience, 15:636124. https://doi.org/doi.org/10.3389/fnhum.2021.636124

Cowen, A. S., \& Keltner, D. (2021). Semantic Space Theory: A Computational Approach to Emotion. Trends in Cognitive Sciences, 25(2), 124-136. 
https://doi.org/10.1016/j.tics.2020.11.004

Furl, N. (2016). Facial-Attractiveness Choices Are Predicted by Divisive Normalization. Psychological Science, 27(10), 1379-1387. https://doi.org/10.1177/0956797616661523

Gelman, A., Lee, D., \& Guo, J. (2015). Stan: A Probalistic Programming Language for Bayesian Inference and Optimization. Journal of Educational and Behavioral Statistics, 40(5), 530-543. https://doi.org/10.3102/1076998615606113

Goldstone, R. L., Medin, D. L., \& Halberstadt, J. (1997). Similarity in context. Memory and Cognition, 25(2), 237-255. https://doi.org/10.3758/BF03201115

Hall, E. T. (1966). The Hidden Dimension. New York: Doubleday.

Henninger, F., Shevchenko, Y., Mertens, U., \& Kieslich, P. (2019). Lab. js: A free, open, online study builder. Psyarxiv.Com.

Iachini, T., Coello, Y., Frassinetti, F., Senese, V. P., Galante, F., \& Ruggiero, G. (2016). Peripersonal and interpersonal space in virtual and real environments: Effects of gender and age. Journal of Environmental Psychology, 45, 154-164. https://doi.org/10.1016/j.jenvp.2016.01.004

Kahneman, D., \& Tversky, A. (1979). Prospect theory: an analysis of decision under risk, 47(2), $263-292$.

Kennedy, D. P., Gläscher, J., Tyszka, J. M., \& Adolphs, R. (2009). Personal space regulation by the human amygdala. Nature Neuroscience, 12(10), 1226-1227. https://doi.org/10.1038/nn.2381

Kobylínska, D., \& Karwowska, D. (2014). Assimilation and contrast effects in suboptimal affective priming paradigm. Frontiers in Psychology, 5, 498. https://doi.org/10.3389/fpsyg.2014.00498

Kontek, K., \& Lewandowski, M. (2018). Range-dependent utility. Management Science, 64(6), 2812-2832. https://doi.org/10.1287/mnsc.2017.2744 
Lloyd, D. M. (2009). The space between us: A neurophilosophical framework for the investigation of human interpersonal space. Neuroscience and Biobehavioral Reviews, 33(3), 297-304. https://doi.org/10.1016/j.neubiorev.2008.09.007

Louie, K., Grattan, L. E., \& Glimcher, P. W. (2011). Reward Value-Based Gain Control: Divisive Normalization in Parietal Cortex. https://doi.org/10.1523/JNEUROSCI.123711.2011

Louie, K., Khaw, M. W., \& Glimcher, P. W. (2013). Normalization is a general neural mechanism for context-dependent decision making. Proceedings of the National Academy of Sciences of the United States of America, 110(15), 6139-6144. https://doi.org/10.1073/pnas. 1217854110

Martin, L. L., Seta, J. J., \& Crelia, R. A. (1990). Assimilation and Contrast as a Function of People's Willingness and Ability to Expend Effort in Forming an Impression. Journal of Personality and Social Psychology, 59(1), 27-37.

Matsumoto, D., \& Sung Hwang, H. (2010). Judging Faces in Context. Social and Personality Psychology Compass, 4(6), 393-402. https://doi.org/10.1111/j.1751-9004.2010.00271.x

Parducci, A. (1965). Category judgment: a range-frequency model. Psychological Review, 72(6), 407-4018.

Quesque, F., Ruggiero, G., Mouta, S., Santos, J., Iachini, T., \& Coello, Y. (2017). Keeping you at arm's length: modifying peripersonal space influences interpersonal distance. Psychological Research, 81(4), 709-720. https://doi.org/10.1007/s00426-016-0782-1

Ruggiero, G., Frassinetti, F., Coello, Y., Rapuano, M., Di Cola, A. S., \& Iachini, T. (2017). The effect of facial expressions on peripersonal and interpersonal spaces. Psychological Research, 81(6), 1232-1240. https://doi.org/10.1007/s00426-016-0806-x

Russell, J. A., \& Fehr, B. (1987). Relativity in the Perception of Emotion in Facial Expressions. Journal of Experimental Psychology: General, 116(3), 223-237. 
https://doi.org/10.1037/0096-3445.116.3.223

Schrammel, F., Pannasch, S., Graupner, S.-T., Mojzisch, A., \& Velichkovsky, B. M. (2009). Virtual friend or threat? The effects of facial expression and gaze interaction on psychophysiological responses and emotional experience. Psychophysiology, 46(5), 922931. https://doi.org/10.1111/j.1469-8986.2009.00831.x

Schwarz, N., \& Bless, H. (2007). Mental Construal Processes: The Inclusion/Exclusion Model Contact information. In D. A. Stapel \& J. Suls (Eds.), Assimilation and contrast in social psychology (pp. 119-141). Philadelphia: Psychology Press.

Siegman, A. W., \& Feldstein, S. (2014). Nonverbal Behavior and Communication (2nd ed.). New York: Psychology Press. https://doi.org/10.4324/9781315799759

Trueblood, J. S., Brown, S. D., Heathcote, A., \& Busemeyer, J. R. (2013). Not Just for Consumers: Context Effects Are Fundamental to Decision Making. Psychological Science, 24(6), 901-908. https://doi.org/10.1177/0956797612464241

Vieira, J. B., Pierzchajlo, S. R., \& Mitchell, D. G. V. (2020). Neural correlates of social and non-social personal space intrusions: Role of defensive and peripersonal space systems in interpersonal distance regulation. Social Neuroscience, 15(1), 36-51. https://doi.org/10.1080/17470919.2019.1626763

Wedell, D. H., Hayes, W. M., \& Kim, J. (2020). Context effects on reproduced magnitudes from short-term and long-term memory. Attention, Perception \& Psychophysics, 1-17. https://doi.org/10.3758/s13414-019-01932-z

Wedell, D. H., \& Parducci, A. (1988). The Category Effect in Social Judgment: Experimental Ratings of Happiness Multisensory Emotion and Cognition View project Context effects in judgment and choice View project. Article in Journal of Personality and Social Psychology, 55(3), 341. https://doi.org/10.1037/0022-3514.55.3.341

Wedell, D. H., Parducci, A., \& Geiselman, R. E. (1987). A formal analysis of ratings of physical 
attractiveness: Successive contrast and simultaneous assimilation. Journal of Experimental Social Psychology, 23(3), 230-249.

Wieser, M. J., \& Brosch, T. (2012). Faces in context: A review and systematization of contextual influences on affective face processing. Frontiers in Psychology, 3, 471. https://doi.org/10.3389/fpsyg.2012.00471 